\title{
AN OPEN TOSS PROBLEM
}

\section{PREM N. BAJAJ and G.R. MENDIETA}

\author{
Department of Mathematics and Statistics \\ The Wichita State University \\ Wichita, KS 67208, U.S.A.
}

(Received January 28, 1992)

\begin{abstract}
We discuss some particular cases of the following problem. Each of $m$ persons tosses a coin. Those who get heads stay for the next round. Those who get tails are eliminated; however, if all persons get tails in a round, they move to the next round (as if each of them had got a head). The problem is to find the possibility that exactly one person is left after $r$ rounds. All coins are considered identical but not necessarily fair.
\end{abstract}

KEY WORDS AND PHRASES. Coin tossing, depletion model. 1991 AMS SUBJECT CLASSIFICATION CODES. Primary, 60C05; Secondary, 62P99.

\section{INTRODUCTION.}

Numerous games are played with players forming a circle. The opening hand decides the sequence (generally clock-wise as seen from above) of players. In each game, the opening hand is either in the most favorable position or most unfavorable position. One natural way to decide the opening hand is to have each person toss a coin and eliminate those who obtain tails, the case of "all getting tails" being considered equivalent to "all getting heads". With a number of players, it is very unlikely that the issue will be decided in one toss by the $m$ players. Hence, the process is repeated, say $r$ times. It will be interesting to find the probability that one person comes out as a winner after $r$ rounds.

This problem was presented by the first author, at the AMS meeting in 1988 in Providence. An interesting paper [1] by Bruss and O'Cinneide presents some aspects, of this problem.

In the following sections, we take up some particular cases of the problem. We invite solution to the general problem.

Throughout $p$ denotes the probability of obtaining a head and $q=1-p$ the probability of getting a tail.

\section{THEOREM.}

Let $P(r, m)$ denote the probability that, starting with $m$ persons, one person wins the toss exactly after $r$ rounds, $m \geq 2, r \geq 1$.

Let $T_{s}=p^{s}+q^{s}, s \geq 2$. Then

$\begin{aligned} \text { (a) } P(r, 2) & =2 p q T_{2}^{r-1} \\ \text { (b) } P(r, 3) & =3 p q^{2} T_{3}^{r-1}+6 p^{3} q^{2} \sum_{\substack{i+t \\ i, j \leq 0, r}} T_{3}^{i} T_{2}^{j} \\ & \end{aligned}$ 
(c) $\quad P(r, 4)=4 p q^{3} T_{4}^{r-1}+12 p^{4} q^{3} \sum_{\substack{i+1, r=2 \\, j \geq 0, r \geq 2}} T_{4}^{i} T_{j}^{j}$

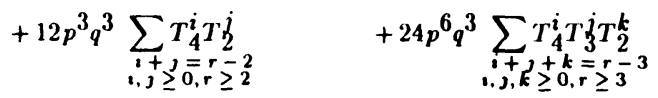

(d) $\quad P(2, m)=m p q^{2 m-1}+m q^{m-1} p^{2}\left[(p+1)^{m-1}-1\right]$.

OUTLINE OF PROOF: Notice that $T_{s}=p^{s}+q^{s}$ is the probability of a tie among $s$ persons (either by all getting tails or all getting heads).

(a) In this case, there is a tie among two players in each of the first $(r-1)$ rounds.

(b) Among three players, game is decided in the $r$ th round in one of the two ways:

(i) There is a three way tie in each of the first $(r-1)$ rounds.

(ii) One player is disqualified sometime in the first $(r-1)$ rounds and another player in the rth round.

In the representation of $P(r, 3)$, first term corresponds to case (i) and the summation to case (ii).

(c) For four players, the game is decided in one of the following ways, depending upon when three players are out.

(i) Three players lose the toss in the last round (and there is a 4-way tie in each of the first $(r-1)$ rounds).

(ii) Two players lose the toss in $r$ th round and one player earlier.

(iii) One player loses in the last round and two players lose earlier either both in the same round or in two separate rounds.

(d) First notice that $P(1, m)=m p q^{m-1}$.

Now

$$
\begin{aligned}
P(2, m) & =\left(p^{m}+q^{m}\right) P(1, m)+\sum_{i=1}^{m-2}\left(\begin{array}{c}
m \\
i
\end{array}\right) p^{m-i} q^{i} P(1, m-i) \\
& =m p q^{2 m-1}+m \sum_{i=0}^{m-2}\left(\begin{array}{c}
m-1 \\
i
\end{array}\right) p^{m-i+1} q^{m-1} \\
& =m p q^{2 m-1}+m q^{m-1} p^{2}\left[(p+1)^{m-1}-1\right] .
\end{aligned}
$$

3. REMARK.

$P(2, m) / P(1, m)=q^{m}+p\left[(1+p)^{m-1}-1\right]$ approaches $+\infty$ with $m$. Thus for large $m$, there is much greater change for the game to be decided in two rounds than in one round.

4. THEOREM.

If the coin is fair $(p=1 / 2)$, then

$$
P(r, m)=m 2^{-r m} \sum_{k=1}^{2^{r}-1}(-1)^{k+1} k^{m-1}
$$

PROOF. For $k=1, \cdots, m$ let $P(r, m, k)$ be the probability that starting with $m$ persons at the end of $r$ rounds there are $k$ players in the game. Then $P(r, m)$ above is just $P(r, m, 1)$.

First note that for $m \geq 2$ and $k=1, \cdots, m$

$$
P(1, m, k)=\left\{\left(\begin{array}{c}
m \\
k
\end{array}\right)+\delta(k-m)\right\}^{-m},
$$


where $\delta(x)$ is the indicator function of $\{0\}$. In particular, we have that.

$$
P(1, m, 1)=m^{2}-m \text {. }
$$

We will use induction to show that (1) holds.

We will show below that if (1) holds for $r=n$ then it also holds for $r=n+1$.

By conditioning on the outcome of the first toss we get that

$$
\begin{aligned}
& P(n+1, m, 1)= \sum_{j=2}^{m} P(1, m, j) P(n, j, 1) \\
&= \sum_{j=2}^{m}\left(\begin{array}{c}
m \\
j
\end{array}\right) 2^{-m} j 2^{-n j} \sum_{k=1}^{2^{n}-1}(-1)^{k+1} k^{j-1}+ \\
& \quad+m 2^{-(n+1) m} \sum_{k=1}^{2^{n}-1}(-1)^{k+1} k^{m-1} \\
&=2-m \sum_{k=1}^{2^{n}-1}(-1)^{k+1} m 2^{-n} \sum_{l=1}^{m-1}\left(\begin{array}{l}
m-1 \\
l
\end{array}\right) k^{l} 2^{-n l}+ \\
& \quad+m 2^{-(n+1) m} \sum_{k=1}^{2^{n}-1}(-1)^{k+1} k^{m-1} \\
&=m 2^{-(n+1) m} \sum_{k=1}^{2^{n+1}}(-1)^{k+1} k^{m-1}
\end{aligned}
$$

after some simplification. This concludes the proof.

\section{REFERENCES}

1. BRUSS, F.T. \& O'CINNEIDE, C.A., On the maximum and its uniqueness for geometric random samples, J. Appl. Prob. 27 (1990), 598-610. 


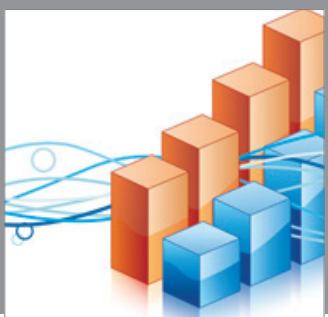

Advances in

Operations Research

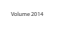

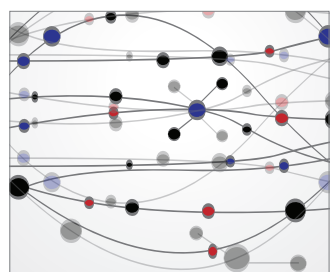

\section{The Scientific} World Journal
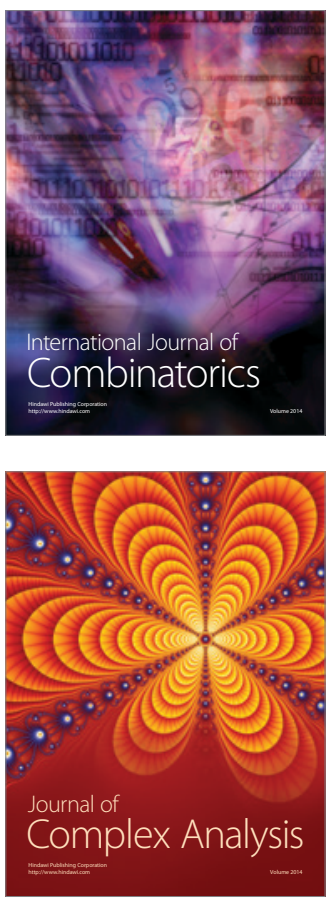

International Journal of

Mathematics and

Mathematical

Sciences
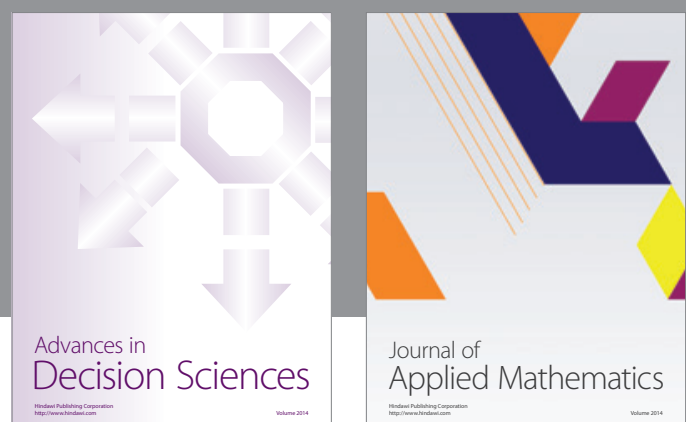

Journal of

Applied Mathematics
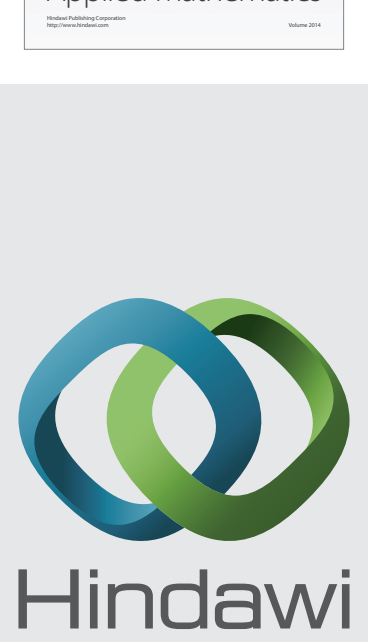

Submit your manuscripts at http://www.hindawi.com
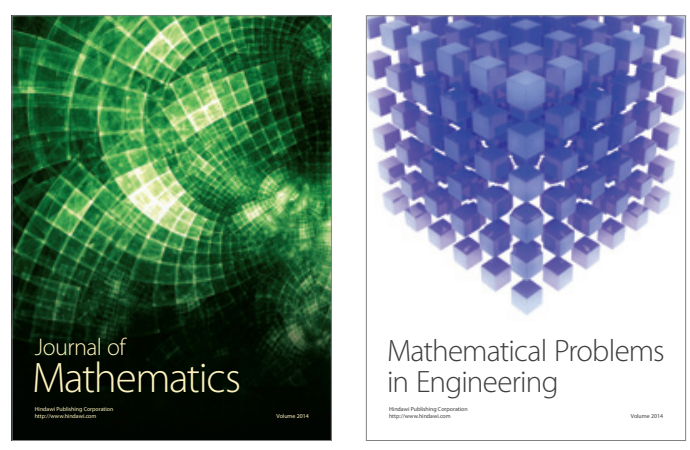

Mathematical Problems in Engineering
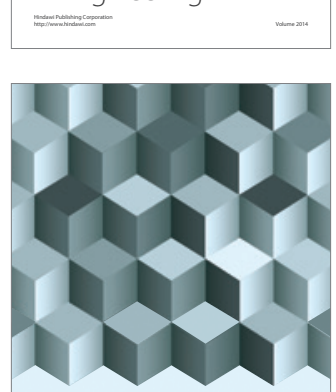

Journal of

Function Spaces
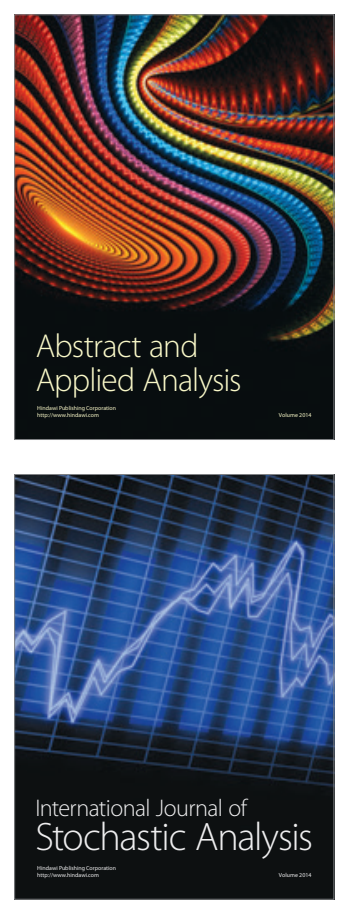

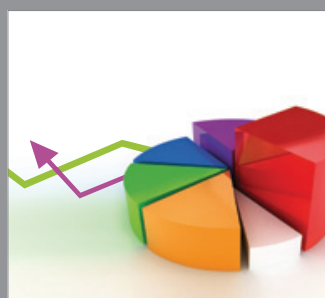

ournal of

Probability and Statistics

Promensencen
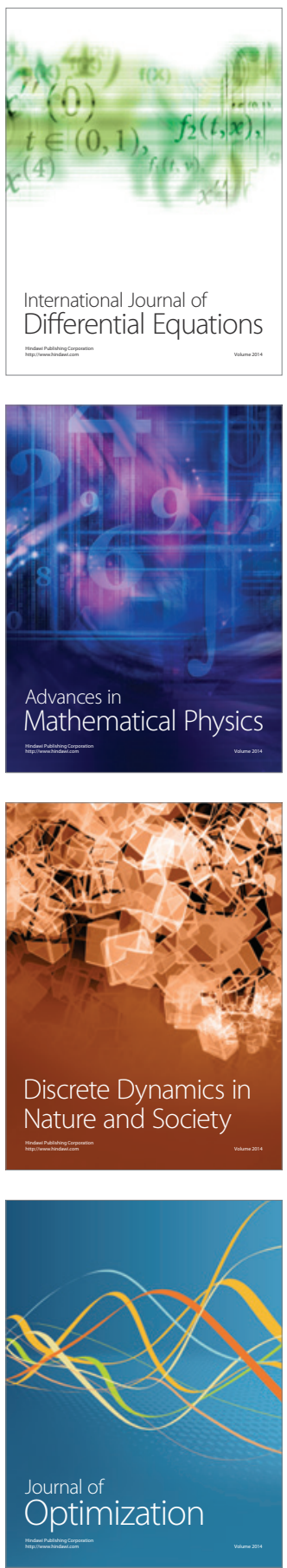